\title{
INFLUÊNCIA DA ANGULAÇÃO DE POÇOS DIRECIONAIS NA VELOCIDADE DE MIGRAÇÃO DE UM KICK
}

\author{
V. F. ALMEIDA ${ }^{1}$, V.F ALMEIDA, K. R. S. FAGUNDES, F. P. FAGUNDES \\ Universidade Potiguar \\ valterlei_f_a@hotmail.com ${ }^{1}$
}

Submetido 06/10/2016 - Aceito 22/02/2017

DOI: $10.15628 /$ holos.2017.5180

\section{RESUMO}

Quando a pressão de poros (Pporos) for superior à hidrostática gerada pelo fluido de perfuração, juntamente com uma alta permeabilidade da formação, possivelmente deverá ocorrer fluxo da formação para o poço ("Kick"). Em caso de um influxo de gás, a pressão de fundo irá aumentar drasticamente e caso não seja escolhido um método de controle adequado, poderá haver enormes riscos operacionais relacionados à perda do poço e, principalmente, à integridade física da equipe de perfuração. Para garantia de operações seguras, é imprescindível que a pressão hidrostática no fundo do poço seja superior a pressão nos poros da formação. Um ponto crítico refere-se à velocidade de migração do kick, onde parâmetros como a geometria do poço, inclinação, vazão, fração de gás e reologia do fluido constituem os principais aspectos a serem considerados. Dessa forma, esse trabalho tem como objetivo principal mostrar os aspectos inerentes ao cálculo de velocidade de migração, levando em consideração a densidade do kick (lb/gal) e do fluido (lb/gal) em associação com a angulação do poço. Em paralelo, foi utilizada uma modelagem fatorial com 3 níveis e 3 variáveis (Statistica 7.0) a fim de mapear o efeito que cada variável exerce sobre a dinâmica do kick. Após plotar as superfícies de resposta, foi possível avaliar estatisticamente o efeito que cada variável exerce sobre a velocidade de migração do kick. Os resultados (diagrama de Paretto) mostraram a forte dependência da velocidade de migração do kick em função da angulação, principalmente, com a inclinação alcançando 45 ․

PALAVRAS-CHAVE: Fluidos de perfuração, pressão de poros, velocidade de migração do kick, poços direcionais e superfície de resposta.

\section{INFLUENCE OF DIRECTIONAL WELL ANGULATION AT THE SPEED OF A KICK MIGRATION}

\begin{abstract}
When the pore pressure (Pores) is greater than the hydrostatic generated by the drilling fluid, in association to high permeability of the formation, will possibly occur flow of formation into the well ("kick"). In case of a gas influx, the bottom pressure will increase dramatically and if a suitable control method is not chosen, there may be huge operational risks related to the loss well, and especially the physical integrity of the drilling crew. To guarantee safe operation, it is essential that the hydrostatic pressure at the bottom is greater than the pressure in the pores of the formation. An critical point refers to migration velocity of kick, where parameters as well geometry, angulation, gas fraction, flow rate and rheological properties of drilling fluids represent the
\end{abstract}

main aspects to be considered. Thus, this work has as main aim to show the inherent aspects to the migration velocity calculus, taking as parameter kick (lb/gal) and fluid density (lb/gal) in association with well angulation. In parallel, it was used a modeling factorial with 3 levels and 3 variables (Software Statistica 7.0) to map the effect that each variable has on the dynamics of the kick. After plotting the response surfaces was statistically possible to assess the effect each variable dictate on kick migration velocity. The results (Paretto diagram) showed the strong dependence of the kick migration velocity as function of well angulation, especially with the inclination reaching $45^{\circ}$.

KEYWORDS: Drilling fluids, pore pressure, kick migration velocidade, directional wells, and response surfasse. 


\section{INTRODUÇÃO}

Nas atividades petrolíferas, os fluidos de perfuração têm importância fundamental. São esses fluidos que devem permitir o resfriamento da broca, a retirada dos cascalhos gerados na perfuração e a manutenção da estabilidade do poço, de acordo com (HANDY, 2011) e (MUKHERJEE, 2013). A geração de uma pressão hidrostática superior à pressão nos poros da formação é uma das funções mais importante do mesmo. Caso a pressão da formação esteja atuando de forma superior sobre a pressão hidrostática e esta dispor de permeabilidade suficiente, ocorrerá migração do fluído (água, óleo ou gás) para o interior do poço.

A deteç̧ão e remoção de um kick devem ser iminentes. Caso exista falha em uma das duas situações, perde-se o controle do fluxo de fluidos, ocasionando um blowout.

Segundo Araújo (2013) a dificuldade para se controlar um kick, cresce com o aumento da diferença entre a pressão de poros da formação e com o aumento do volume de fluido que invade o poço. Para Nas (2011), uma rocha com alta permeabilidade e porosidade tem maior potencial para ocorrência de um kick como, por exemplo, a rocha arenito.

De acordo com Otto (2013), durante a perfuração existem indícios da formação de um kick como o aumento do volume de fluido nos tanques, aumento da vazão de retorno, fluxo de fluido com as bombas desligadas, poço "aceitando" um volume de fluido menor que o do volume de aço retirado e devolvendo mais fluido que o volume do aço introduzido. Baseado nesses cenários a estimativa da velocidade de migração do gás no fluido de perfuração deve ser ressaltada, a mesma é em função de algumas características como: pressão do poço, perfil de temperatura do fluido, fator volume-formação, vazão do kick, volume de gás dissolvido no poço, vazão de retorno e ganho no tanque de fluido. A justificação para isso é que a ascensão do gás no fluído de perfuração é abstrusa por depender de vários fatores e circunstâncias como: tamanho e distribuição da bolha de gás, reologia do fluido de perfuração, geometria do espaço anular, pressão do gás e, principalmente inclinação do poço conforme (NAS, 2011).

Dessa forma, constitui um desafio-chave avaliar a migração do gás em poços inclinados ao método de controle de kick e associar ambos os parâmetros à inclinação do poço. Por essa razão, o objetivo desse trabalho consiste em avaliar a velocidade de migração do kick por correlações matemáticas (literatura), levando em consideração a densidade do kick (lb/gal) e do fluido (lb/gal) em associação com a angulação do poço. Em paralelo, utilizar uma modelagem fatorial (Statistica 7.0) a fim de mapear o efeito que cada variável exerce sobre a dinâmica do kick.

\section{REVISÃO BIBLIOGRÁFICA}

\subsection{Influência do fator de compressibilidade do fluido na dinâmica do kick}

O comportamento de bolhas em fluidos não-newtonianos é de fundamental importância em diversos setores. O conhecimento da hidrodinâmica, em particular a velocidade de ascensão das bolhas é essencial para avaliar o deslocamento do kick e o método a ser utilizado para o seu controle em poços de petróleo. O intervalo de tempo entre o início do kick e a detecção do mesmo é uma informação primordial para inferir o seu volume. Se o mesmo for detectado rápido, o volume de influxo que entrou no poço é reduzido e, consequentemente, a máxima 
pressão que ocorrerá em uma dada localização dentro do poço também é menor, facilitando as operações de controle de poço e reduzindo o risco de blowout.

Silva et al. (2004) avaliou a solubilidade do gás em fluidos sintéticos utilizados em perfurações HPHT (altas temperaturas e altas pressões) em águas profundas na Bacia de Campos, Brasil. De acordo com os resultados obtidos, nas temperaturas de $158{ }^{\circ} \mathrm{C}$ e $194{ }^{\circ} \mathrm{C}$ foi verificado que fluido n-parafina apresenta uma maior solubilidade de gases, o que certamente, em virtude dos parâmetros reológicos e taxa de compressibilidade torna mais difícil sua detecção (Ver figura 1). Esse fato pode ser perfeitamente observado na figura 1, onde a expansão de 10 barris de fluido de base n-parafina ao longo de um poço de 4800 metros de acordo com (GALVES, 2013).

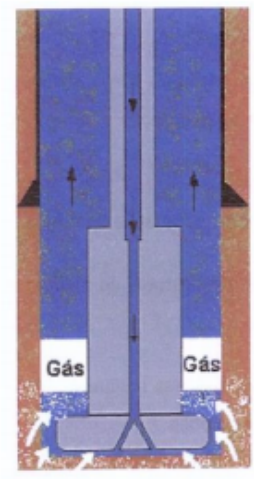

Fluido Base Água

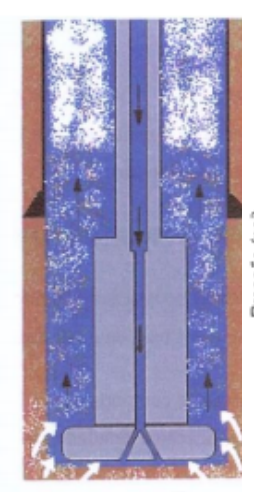

Fluido Base Óleo

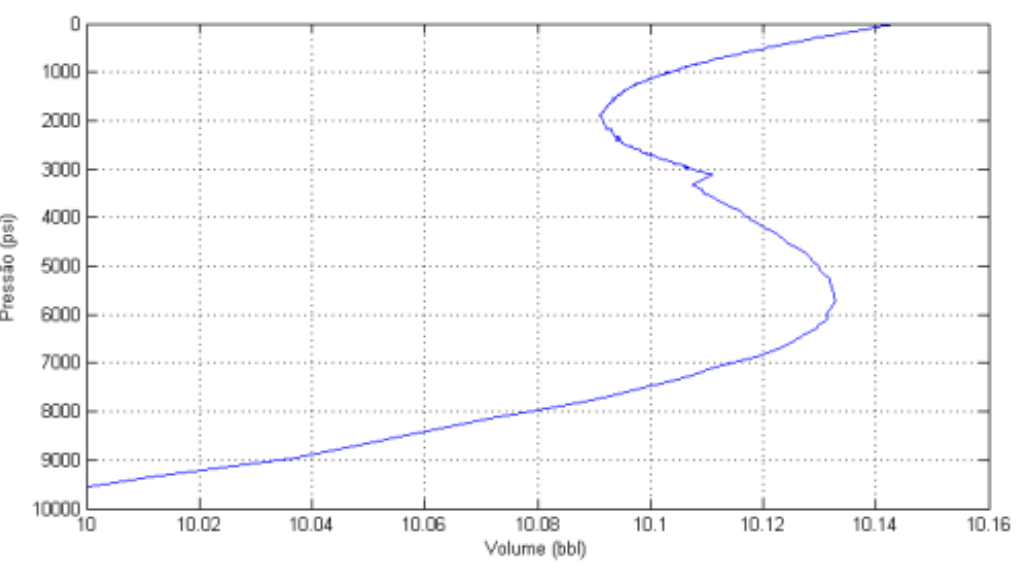

Figura 1: Expansão de 10 barris de fluido de base $n$-parafina ao longo de um poço de 4800 metros.

Galves (2013) utilizou um programa em Matlab que considerou correlações para razão de solubilidade e fator volume de formação obtidas da literatura, um modelo de reservatório radial, escoamento bifásico em golfada e transferência de calor da formação para o fluido. $O$ gás estudado foi o metano e o fluido de perfuração, de base n-parafina. De acordo com resultados ficou claramente observado que a detecção de kick em fluidos à base de n-parafina é menos perceptível que em fluidos aquosos (ver figura 2). 


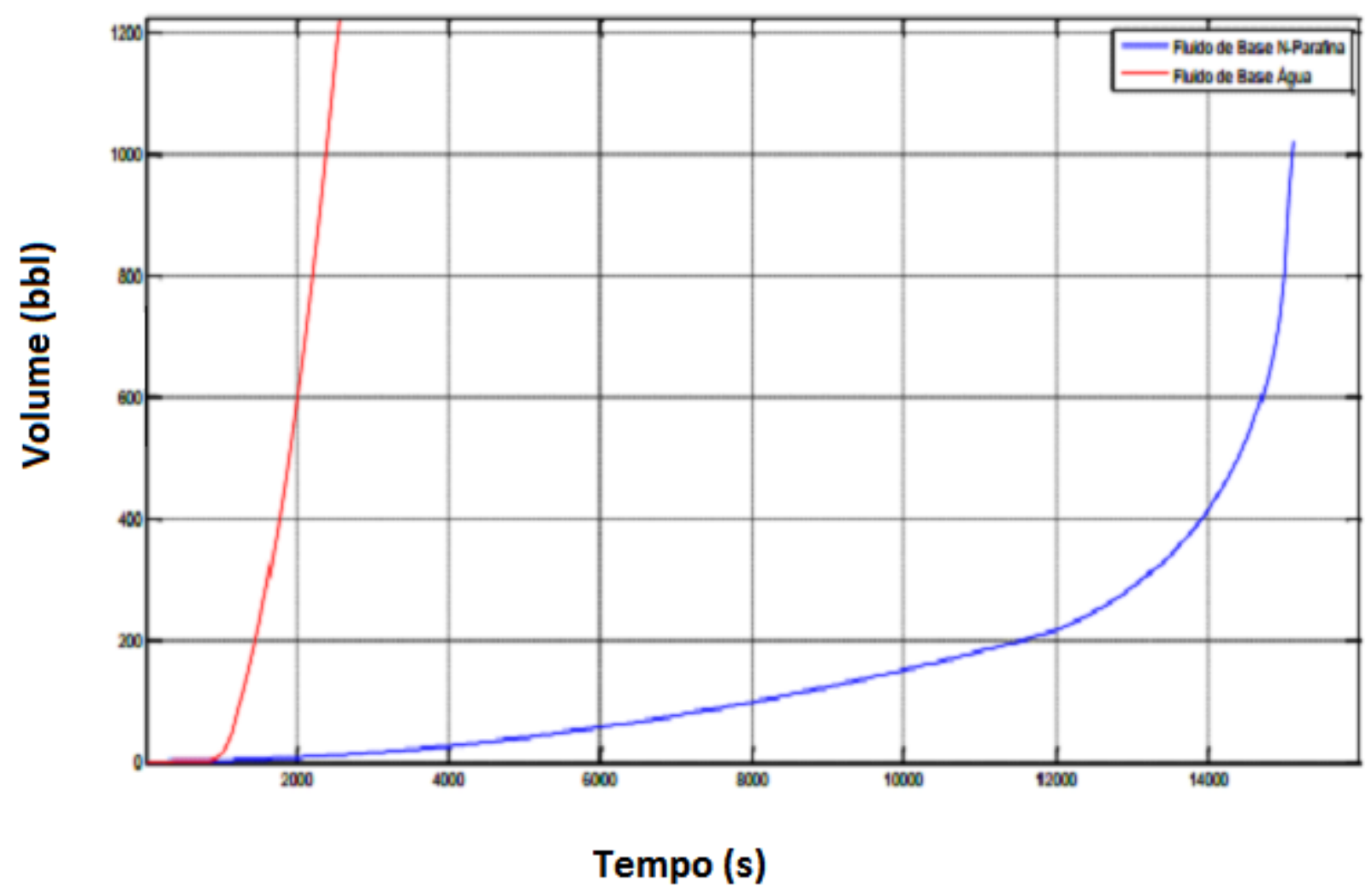

Figura 2: Ganho de volume no tanque no cenário-padrão proposto por Galves.

Dessa forma, existe uma forte dependência da velocidade de migração do kick ao longo do seu deslocamento com os parâmetros reológicos e taxa de compressibilidade, tornando-se imprescindível avaliação dos mesmos para o controle da hidrostática do poço.

\subsection{Fatores que afetam a velocidade de migração do kick}

Tomando-se como de início a dinâmica da bolha de gás isolada é possível estudá-la. 0 comportamento da bolha de gás pode ser descrita por um conjunto de equações que inclui a equação do movimento da bolha, as equações da continuidade e movimento para o fluido a condição de aderência na interface fluido-partícula e mais as equações constitutivas para o fluido e as condições limites pertinentes ao problema específico segundo (C.R. STOKES, 1850). A análise limita-se à dinâmica da bolha em fluidos newtonianos e pseudoplásticos, e há a necessidade de conhecimento prévio da reologia do fluído e das propriedades físicas da bolha de gás.

Segundo Weisman e Kang (1981) o escoamento de gás e líquido em uma tubulação assume diferentes arranjos espaciais dependentes das velocidades das duas fases, chamados de padrões de escoamentos. A maioria dos padrões de escoamentos descritos na literatura é comum a todos os ângulos de inclinação da tubulação, porém existem exceções, onde, de acordo com o ângulo de inclinação, algumas características de um mesmo regime se modificam.

Krishna et al. (2000) avaliaram a velocidade de ascensão de bolhas de ar em fluido Newtoniano (água). Com a variação do diâmetro da bolha de $3-80 \mathrm{~mm}$ em associação com o diâmetro da coluna para avaliar o efeito de parede, foi observado que o diâmetro da coluna tem 
um efeito significativo sobre a velocidade de ascensão da bolha. Além disso, foi verificado que a razão do diâmetro da bolha/diâmetro de coluna é menor que 0.125.

Terasaka e Tsuge (2001) realizaram estudos aplicando diferentes condições para o experimento, tais como volume da câmara de gás, diâmetro interno da coluna, vazão de gás e parâmetros reológicos do fluido, na formação da bolha. Utilizando fluidos viscoplásticos, como goma xantana e diferentes concentrações de solução de Carbopol, verificaram que o volume da bolha aumenta com os parâmetros citados. Ademais, estabeleceram um modelo para formação de bolha não esférica em fluidos com comportamento modelado pela equação de HerschelBulkley.

Para bolhas de gás em ascensão que possuem densidades inferiores ao do fluido em que se encontra, possuem baixa inércia mecânica e o desequilíbrio das forças pode resultar em trajetórias espiraladas onde há a variação da amplitude e períodos em função da densidade, diâmetro da bolha e angulação do poço (Ver Figura 3).

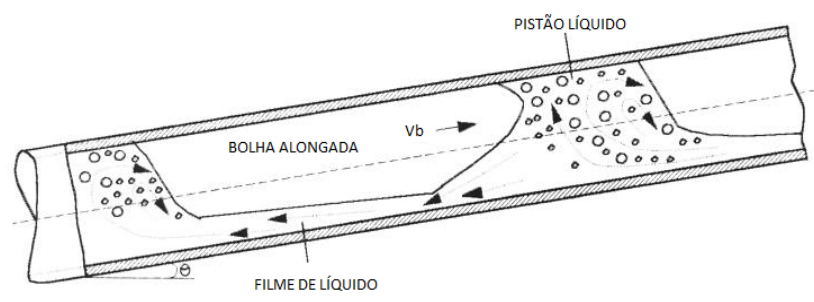

Figura 3: Deslocamento de um kick de gás no anular de um poço inclinado.

Estudos realizados por Luewisutthichat (1997) constataram que o movimento da bolha está relacionado com a deformação na sua forma. Enquanto bolhas com formatos esféricos apresentaram trajetórias relativamente planas, as bolhas com formato elipsoidal e calota esférica demonstraram trajetórias irregulares em forma de zig-zag.

Segundo Dumitrescu (1943) em escoamento vertical, a bolha apresenta a característica um nariz esférico e uma fina camada de líquido simétrica, que separa a bolha da parede do tubo, chamada de filme de líquido (Ver Figura 1). Conforme ocorre a inclinação do tubo, o filme de líquido perde a simetria e o nariz perde sua forma esférica. No escoamento horizontal, a bolha concentra-se na metade superior do tubo e possui um nariz assimétrico conforme (VINHAS, 2015).

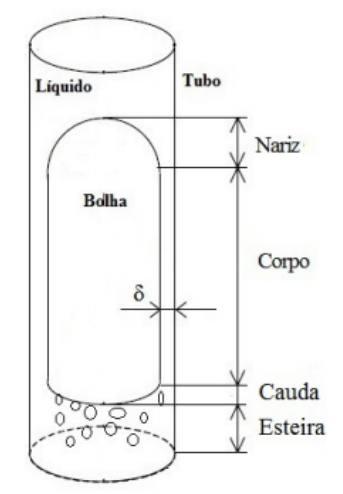

Figura 4: Esquema de uma bolha de Taylor. 
Uma das dificuldades relacionadas aos trabalhos envolvendo escoamentos multifásicos tem a ver com os arranjos geométricos das fases, que são extremamente complexos e se encontram longe de serem bem compreendidos conforme (SHOHAM, 2006). Uma distribuição particular da geometria das fases é denominada padrão de escoamento de acordo com (GALVES, 2013). O escoamento de gás e líquido em uma tubulação assume diferentes arranjos espaciais dependentes das velocidades das duas fases, chamados de padrões de escoamentos, onde são fortemente dependentes do ângulo de inclinação do poço segundo (VINHAS, 2015), como pode ser visualizado na Figura 5.

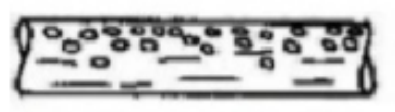

Escoamento em Bolhas

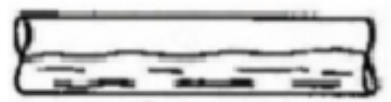

Estratificado Ondulado

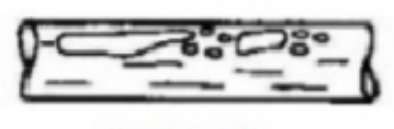

Pistonado

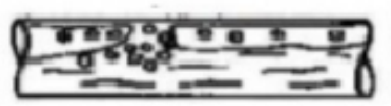

Slug

(a)

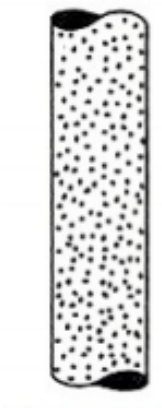

Escoamento em Bolha

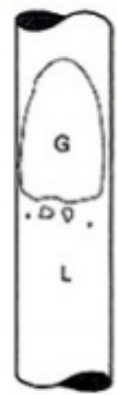

Slug

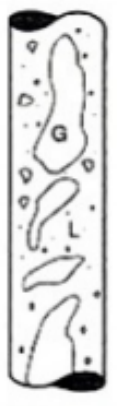

Churn

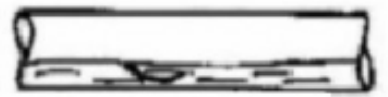

Estratificado Suave

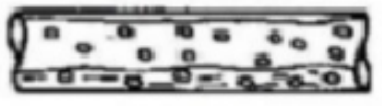

Anular

(b)

Figura 5: Padrões de escoamento líquido/gás.

O regime adotado nesse trabalho foi de escoamento em golfadas, onde o mesmo é caracterizado por ser simétrico com relação ao eixo do duto. A fase gasosa é bem pronunciada e a fase líquida é contínua. A maior parte do gás se concentra em bolsas de tamanho expressivo, conceitualmente conhecida como bolhas de Taylor. Consiste em uma sucessão de bolhas e golfadas líquidas que cobre toda a seção transversal do duto. Nesse tipo de escoamento, a velocidade das bolhas de gás é maior do que a do líquido; tanto gás, quanto líquido, ambos representem significativa influência no gradiente de pressão (Ver Figura 3 - escoamento Slug). 


\section{METODOLOGIA}

Segundo Otto (2013) existe uma complexidade para se estimar a velocidade de migração de uma bolha de gás em um fluido de perfuração tendo em vista a dependência de vários parâmetros como:

- O tamanho e distribuição da bolha de gás;

- A reologia do fluido, geometria do espaço anular;

- Inclinação do poço, pressão do gás e a densidade dos fluidos.

Por essa razão, foram adotadas correlações matemáticas sequenciais (Equação 1, 2 e 3) da literatura com o intuito de avaliar o efeito da densidade do kick, densidade do fluido e a angulação do poço em relação à velocidade de migração de bolhas de gás, associando o efeito de cada variável à dinâmica do kick em poços de petróleo (HASAN \& KABIR, 1992).

$V_{T B \theta}=V_{T B} \sqrt{\sin \theta}(1+\cos \theta)^{1.2}$

$V_{T B \theta_{a}}=\left[0.345+0.1 \frac{D_{i}}{D_{o}}\right] \sqrt{\left(\frac{g D_{o}\left(\rho_{L}-\rho_{G}\right)}{\rho_{L}}\right)}$

$V_{\text {TB } \theta_{a}}=\left[0.345+0.1 \frac{D_{i}}{D_{o}}\right] \sqrt{\sin \theta}(1+\cos \theta)^{1.2} \sqrt{\left(\frac{g D_{o}\left(\rho_{L}-\rho_{G}\right)}{\rho_{L}}\right)}$

Onde: Di e D0 representam os diâmetros interno do poço e externo da coluna; pL e pg as densidades do fluido de perfuração e do fluido invasor, respectivamente; $\varphi$ a angulação do poço, g a gravidade $(\mathrm{m} / \mathrm{s} 2)$ e VTB $\varphi$ a é a velocidade de migração do kick $(\mathrm{m} / \mathrm{s})$.

O efeito de cada variável foi analisado em função de um mesmo cenário, com os mesmos parâmetros, possibilitando assim, o efeito comparativo em função de cada uma das variáveis estudadas separadamente. As faixas de variação de cada parâmetro são apresentadas na tabela 1.

Tabela 1: Níveis das variáveis estudadas.

\begin{tabular}{c|c}
\hline Variáveis & Intervalos \\
$\begin{array}{c}\text { Angulação (graus) } \\
\text { Massa específica do } \\
\text { fluido (lb/gal) }\end{array}$ & $0-90$ \\
$\begin{array}{c}\text { Massa específica do kick } \\
\text { (lb/gal) }\end{array}$ & $1,3-8,33-13$ \\
\hline
\end{tabular}




\subsection{Metodologia de superfície de resposta}

Superfícies de respostas são utilizadas para examinar as relações entre uma ou mais variáveis e um conjunto quantitativo de fatores experimentais. Esses métodos estatísticos são empregados após uma triagem dos fatores importantes, que geralmente é realizada previamente por um planejamento fatorial. Após isso, é preciso encontrar um dos níveis de fatores que otimizem a resposta. Para estudar a otimização das variáveis inerentes à velocidade de migração do kick, a angulação do poço e as densidades do fluido invasor e do fluido de perfuração foram avaliadas (Equação 3). O planejamento fatorial de $3^{3}$ foi utilizado: três níveis com três efeitos principais associados à densidade do fluido, do kick e da angulação do poço, conforme pode ser visualizado na Tabela 2.

Tabela 2: Planejamento fatorial adotado para a determinação dos efeitos.

\begin{tabular}{|c|c|}
\hline Variáveis & Intervalos \\
\hline Angulação (graus) & $\begin{array}{c}0(-)-45(0)-90 \\
(+)\end{array}$ \\
\hline $\begin{array}{l}\text { Massa específica do } \\
\text { fluido (lb/gal) }\end{array}$ & $\begin{array}{c}8,33(-)-10-, 5(0) \\
-13(+)\end{array}$ \\
\hline $\begin{array}{c}\text { Massa específica do kick } \\
\text { (lb/gal) }\end{array}$ & $\begin{array}{c}1,3(-)-5(0)- \\
8,33(+)\end{array}$ \\
\hline
\end{tabular}

Os níveis dos fatores foram codificados como -1 (mínimo), 0 (médio) e 1 (máximo). Para tratamento dos dados, o programa estatístico Statistic Software versão 7.0 foi empregado para obtenção dos efeitos, coeficientes e desvio padrão dos coeficientes, gráficos dos efeitos padronizados, gráfico de pareto e as superfícies de respostas e de outros parâmetros estatísticos dos modelos ajustados.

\subsection{Hipóteses}

As hipóteses e considerações foram executadas de modo a, muitas vezes, simplificar o código e o tornar factível do ponto de vista teórico-computacional, sem o comprometimento da credibilidade dos resultados. Dentre os quais destacam-se:

$\checkmark$ Fluido de perfuração com reologia segundo o modelo da Lei de Potências;

$\checkmark$ Solubilização total e instantânea do gás;

$\checkmark$ Gás que sai de solução empurra o fluido de perfuração acima;

$\checkmark$ Angulação do poço variando de 10 - 90 ; 
$\checkmark$ Produção de cascalhos insuficiente para alterar a densidade do fluido de perfuração ou para ter qualquer implicação sobre o escoamento;

$\checkmark$ Escoamento em golfada.

\section{RESULTADOS E DISCURSSÕES}

Com base na equação proposta por Hassan e Kabir (1992) foram avaliados os efeitos relacionados à densidade do fluido, do kick e a angulação do poço, levando em consideração as hipóteses mencionadas na seção experimental. Em paralelo, através de uma modelagem fatorial com 3 níveis e 3 variáveis $\left(3^{3}\right)$ foi possível mapear através de MSR (Metodologia de Superfície de Resposta) as interações dessas variáveis no processo de migração do kick em um poço de petróleo.

4.1 Influência da massa específica do fluido em função da velocidade de migração do kick.

A Figura 6 abaixo representa os resultados obtidos para a relação entre massa específica do fluído e a velocidade de migração do kick.

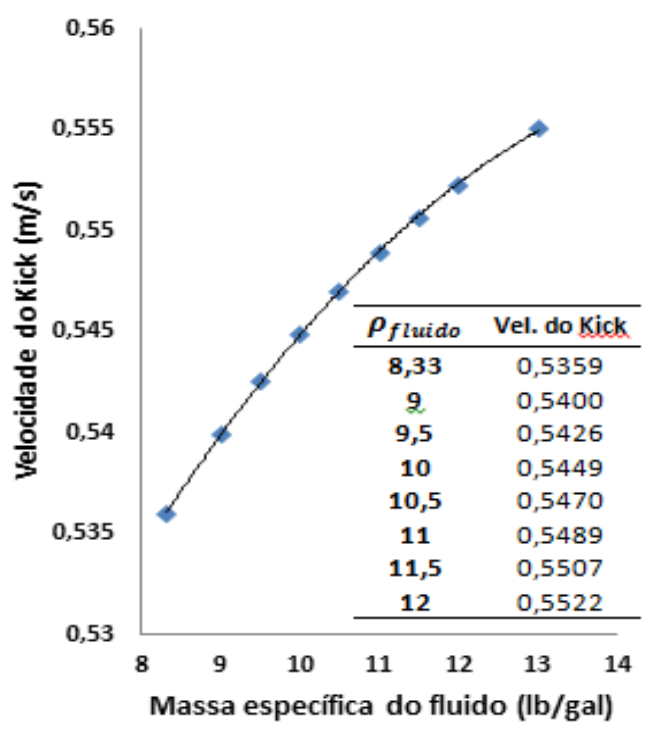

Figura 6: Velocidade do Kick em função da massa específica do fluído.

Observar-se na figura 6 acima um acrécimo da velocidade do kick em meio ao aumento da massa específica do fluído, isso ocorre porquê a ascensão de uma bolha através de um líquido mais denso em repouso é governada pela interação entre as forças de empuxo, gravitacional e outras forças que atuam na bolha como resultado de sua forma e movimento, quanto maior for a densidade do fluido, maior o empuxo na bolha de gás. 
4.2 Influência da massa específica do kick em função da sua velocidade de migração.

A figura 7 abaixo representa os resultados obtidos para a relação entre massa específica do kick e a velocidade de migração do kick.

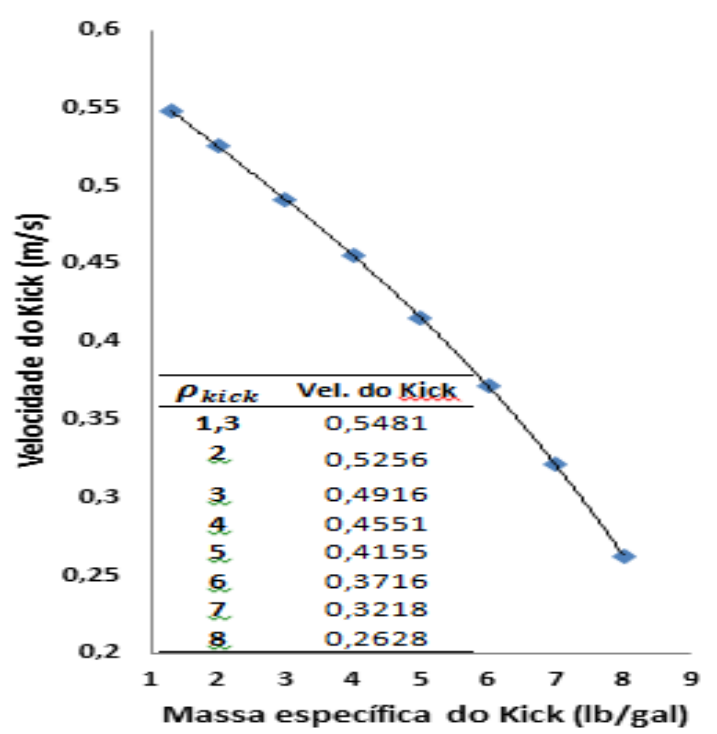

Figura 7: Velocidade do Kick em função da massa específica do kick.

Neste caso, o que é observado na figura 7 é que à medida que se aumenta a massa específica do kick, observa-se um decréscimo na velocidade do mesmo, tendo em vista que a densidade do fluído diminui perante a da bolha de gás, então o kick acaba recebendo menos empuxo.

4.3 Influência da massa específica do fluido em função da velocidade de migração do kick.

A figura 8 mostra o comportamento da velocidade de migração do kick em função da angulação do poço.

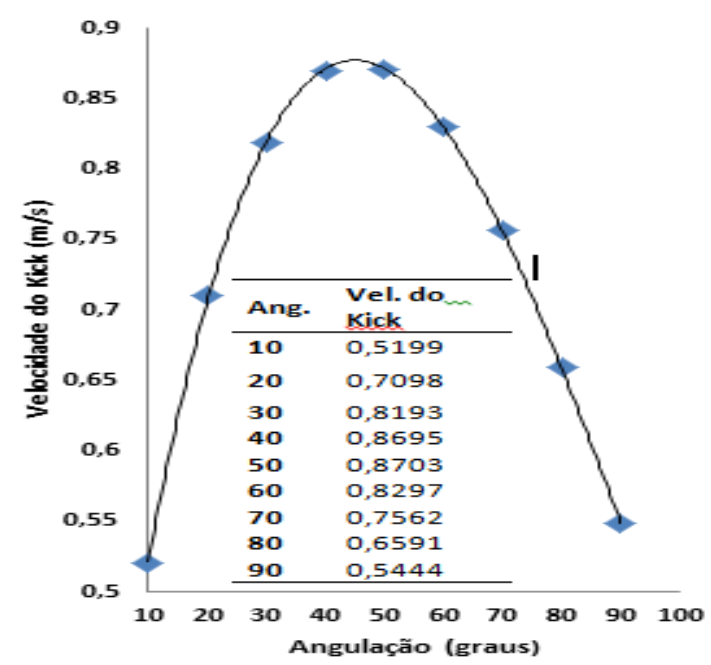

Figura 8: Velocidade do Kick em função da angulação. 
De acordo com a curva do gráfico na figura 8, é observado um maior incremento de velocidade em torno de $45^{\circ}$. A razão para esse efeito está ligada as tensões superficiais em torno do kick de gás e a geometria da bolha. Zukoski (1966) estudou a influência da viscosidade, da tensão superficial e da inclinação no movimento de bolhas alongadas em tubos fechados e aplicou Reynolds (Re) para avaliar a influência da viscosidade e o parâmetro de tensão superficial $(\Sigma)$, como observado na equação 5 , que também estabelece uma relação com a equação de Eotvos. Da mesma forma, Zukoskl (1966) utiliza o termo velocidade normalizada da bolha (Velocidade do equilíbrio em função dos efeitos de parede do sistema), para descrever o número adimensional da equação 6.

$$
\begin{aligned}
& \Sigma=\sigma /\left(\Delta \rho g R^{2}\right)=4 / E o \\
& =W b / \sqrt{ }\left(\frac{\Delta \rho}{\rho L} g R^{2}\right)
\end{aligned}
$$

Onde $\sigma \mathrm{L}$ a tensão superficial, $\Delta \rho$ a diferença de densidade entre o líquido e o gás, $g$ a aceleração da gravidade e $R$ o raio interno do tubo, Wb a velocidade de ascensão da bolha (velocidade de migração do gás/kick) e $\rho L$ a densidade do líquido, Eo é o número de eotvos.

Baseado nas equações 5 e 6, Zukoski, obteve o gráfico observado na figura 9, com isso observou-se que a velocidade de ascensão da bolha de gás era maior quando as tensões superficiais sobre a mesma era menor. Isso era explicado por meio da equação 7 de Eotvos, segundo Vinhas (2015) é possível relacionar o efeito da força de empuxo com a tensão superficial que atua no fluido, ou seja, quanto menor a tensão superficial, maior era a força de empuxo aplicada sobre a bolha de gás.

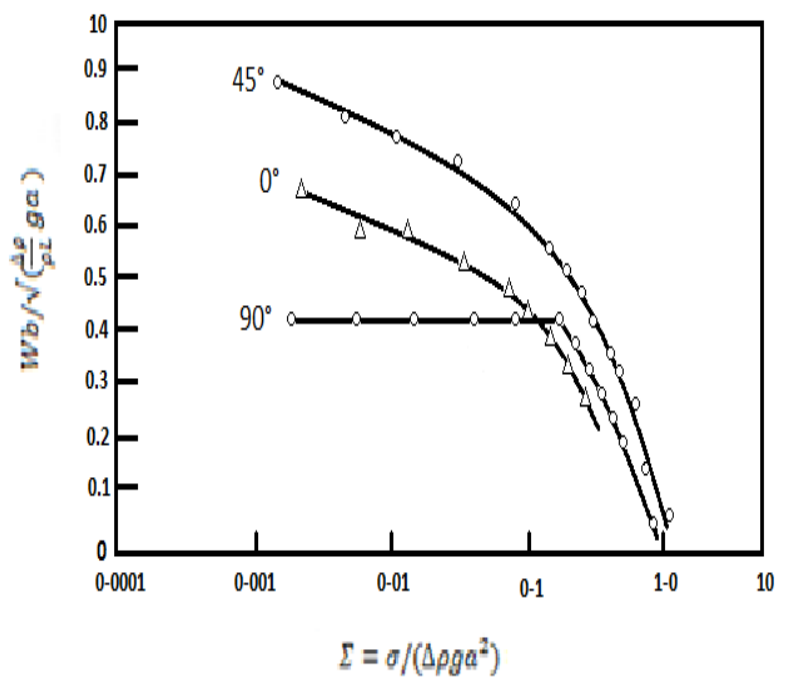

Figura 9: Influência do parâmetro de tensão superficial para os ângulos de inclinação.

$E o=\left(\Delta \rho g D_{i}^{2}\right) / \sigma l$ 
Onde $\sigma \mathrm{L}$ a tensão superficial, $\Delta \rho$ a diferença de densidade entre o líquido e o gás, $g$ a aceleração da gravidade e $R$ o raio interno do tubo, Wb a velocidade de ascensão da bolha e $\rho \mathrm{L}$ a densidade do líquido, Eo é o número de eotvos.

A figura 9 representa a relação que existe entre a tensão superficial e a velocidade de ascensão de uma bolha de gás para angulações de $0^{\circ}, 45^{\circ}$ e $90^{\circ}$.

A velocidade ser maior em $45^{\circ}$ é explicado pelo fato de que quando o ângulo de inclinação do poço aumenta, a bolha tende a ocupar o lado alto do poço, deixando uma área maior para o fluido descendente escoar, aumentando assim a velocidade de migração. Acima de $45^{\circ}$ a velocidade diminui em razão da virtude da redução das forças de interação entre bolha e o fluido em que ele esta imerso tendo em vista a mudança da geometria da bolha. Em $90^{\circ}$, não há migração e sim uma elongação da bolha causada pela tendência em ocupar o lado alto do poço.

\subsection{Metodologia de superfície de resposta}

A Figura 10 representa a superfície de resposta da velocidade de migração do kick $(\mathrm{m} / \mathrm{s})$ em função densidade do kick e da densidade do fluido.

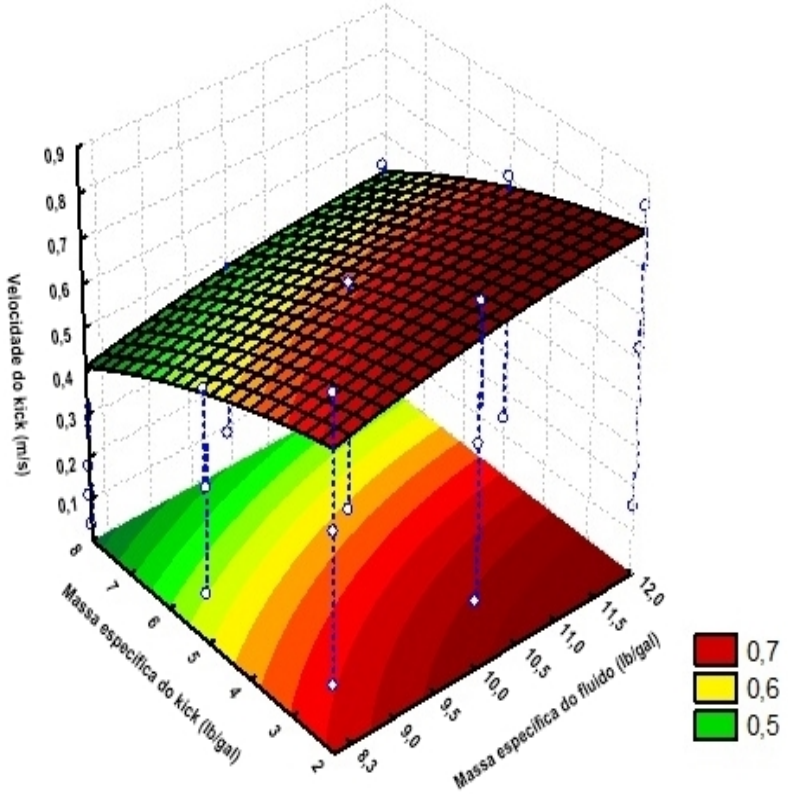

Figura 10: Velocidade do kick em função das densidades do kick e do fluido.

De acordo com a superfície de resposta obtida da Figura 10, é possível observar na zona vermelha a região com maiores velocidade de migração do kick em função das variáveis estudadas, densidade do fluido e do kick. O mapeamento indica que a densidade do fluido representa uma maior contribuição frente à velocidade de migração. Dessa forma, é observado que quanto maior for o diferencial de pressão (densidade) maior será a velocidade de migração. Obviamente que esse comportamento pode ser mudado em virtude de demais fatores como: angulação, geometria do poço, tamanho e formato de bolha.

A figura 11 mostra a interação de parâmetros entre a angulação, massa específica do fluido (lb/bbl) e a velocidade da bolha de gás relacionada à velocidade de migração. A zona 
vermelha é a região com mais velocidade de migração do kick. De acordo com o mapeamento realizado, ficou comprovado que a angulação de 45 graus é responsável por ditar a maior velocidade de migração do kick quando relacionado com densidade do fluído.

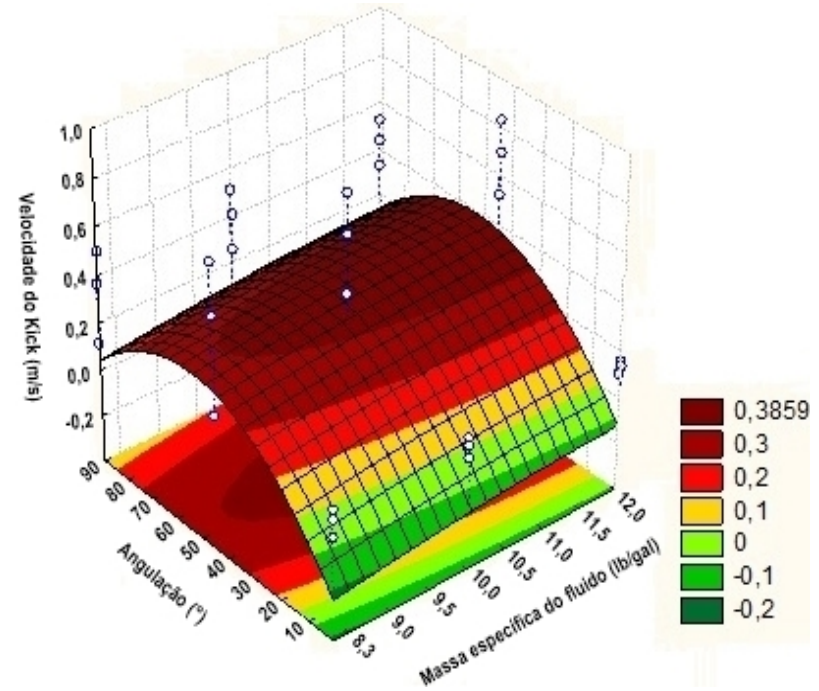

Figura 11: Velocidade do kick em função das densidades do fuido e da angulação.

A figura 12 mostra a interação de parâmetros entre a angulação, massa específica do kick (lb/bbl) e a velocidade da bolha de gás relacionada à velocidade de migração. A zona vermelha é a região com mais velocidade de migração do kick. De acordo com o mapeamento realizado, ficou comprovado que a angulação de 45 graus é responsável por ditar a maior velocidade de migração do kick quando relacionado com densidade da bolha.

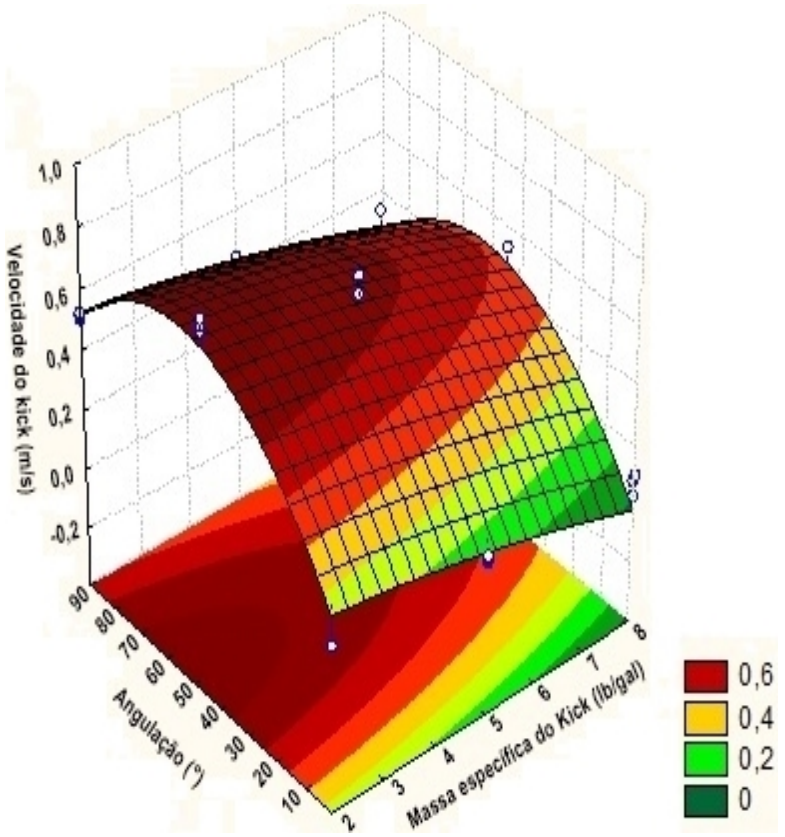

Figura 12: Velocidade do kick em função das densidades do kick e da angulação. 
A figura 13 mostra o diagrama de Pareto para as variáveis analisadas.

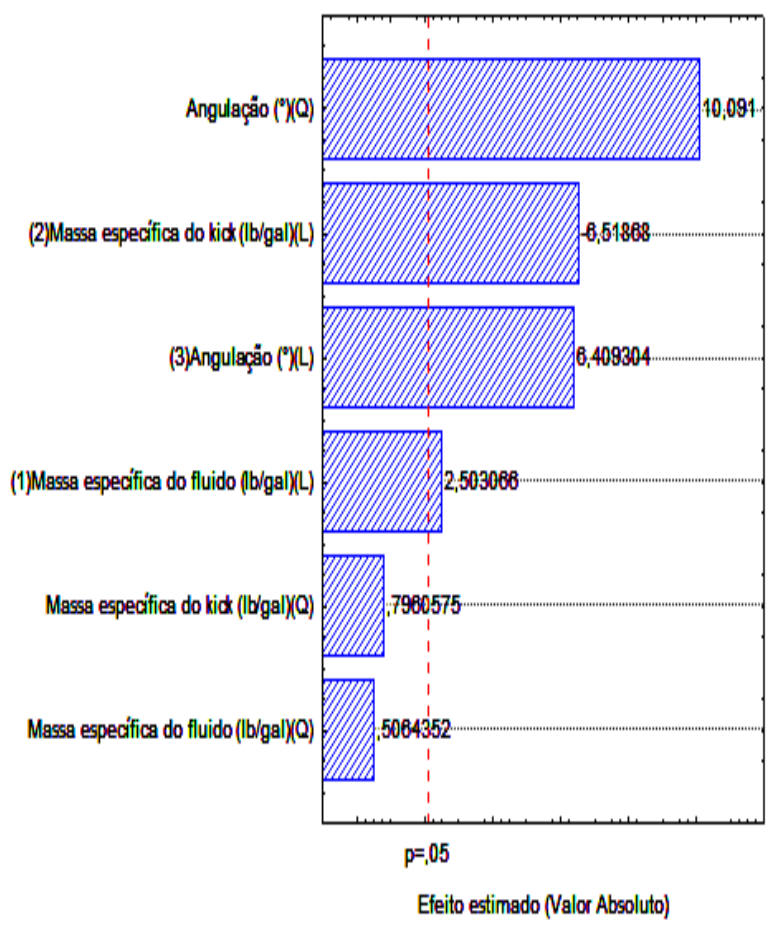

Figura 13: Gráfico de Pareto evidenciando análises das variáveis.

Observou-se que a angulação exerceu uma maior significância estatística no modelo linear e quadrático comparada às demais variáveis.

\section{CONCLUSÃO}

De acordo com a pesquisa realizada foi possível constatar que a angulação de 45 graus apresentou uma forte influência na velocidade de migração da bolha de gás. A existência de maiores diferenciais de densidade entre o fluido e o kick resultou em velocidades de migração também maiores. A tensão superficial é uma das variáveis que influenciam diretamente na velocidade de migração do kick tendo em vista sua relação direta com equação de Eotvos que é uma relação inversamente proporcional da força de empuxo com a tensão superficial. 0 mapeamento da superfície de resposta representou uma ferramenta importante no delineamento da dinâmica de migração da bolha no fluido

\section{REFERÊNCIAS}

Azevedo, M. B., Faccini, J. L., \& Su, J. (2015). Experimental study of single taylor bubbles rising in stagnant liquid mixtures inside of vertical tubes. In Proceedings of the 2015 International Nuclear Atlantic Conference-INAC2015. Disponível em: http://www.iaea.org/inis/collection/NCLCollectionStore/_Public/46/135/46135087.pdf 
Dumitrescu, D. T. (1943). Strömung an einer Luftblase im senkrechten Rohr. ZAMM-Journal of Applied Mathematics and Mechanics/Zeitschrift für Angewandte Mathematik und Mechanik, 23(3), 139-149. Retrieved from doi: 10.1002/zamm.19430230303

Galves, L. V. (2013). Impacto da solubilidade de gás na deteç̧ão de kicks em fluidos de perfuração de base n-parafina. Universidade Federal do Rio de Janeiro

Handy, R., \& Spangler, M. (2007). Geotechnical engineering: soil and foundation principles and practice. (5th Ed.). New York: McGraw Hill Professional.

Hasan, A. R., \& Kabir, C. S. (1992). Two-phase flow in vertical and inclined annuli. International Journal of Multiphase Flow, 18(2), 279-293. Retrieved from doi: 10.1016/0301-9322(92)90089-Y

Kouba, G. E., Wang, S., Gomez, L. E., Mohan, R. S., \& Shoham, O. (2006, January). Review of the State-of-the-Art Gas-Liquid Cylindrical Cyclone (GLCC) Technology-Field Applications.

In International Oil \& Gas Conference and Exhibition in China. Society of Petroleum Engineers.

Krishna, R. et al. (2000). Three-phase Eulerian simulations of bubble column reactors operating in the churn-turbulent regime: a scale up strategy. Chemical Engineering Science, 55(16), 3275-

3286. Retrieved from doi: 10.1016/S0009-2509(99)00582-5

Luewisutthichat, W., Tsutsumi, A., \& Yoshida, K. (1997). Bubble characteristics in multi-phase flow systems: bubble sizes and size distributions. Journal of chemical engineering of Japan, 30(3), 461-466. Retrieved from doi: 10.1016/j.cherd.2008.04.006

Mukherjee, S. (2013). The science of clays. Applications in Industry, Engineering and Environment. (1st Ed.). India: Springer.

Nas, S. (2011, January). Kick Detection and Well Control in a Closed Wellbore. In IADC/SPE Managed Pressure Drilling and Underbalanced Operations Conference \& Exhibition. Society of Petroleum Engineers.

Otto L. A. S. (2013). Segurança de poço na perfuração. (1ST Ed.). São Paulo: Blucher.

Silva, C. T. et al. (2004). Estudo da solubilidade de metano em liquidos organicos utilizados na formulação de fluidos de perfuração. Tese de mestrado, Universidade Estadual de Campinas, Campinas, SP, Brasil.

Terasaka, K., \& Tsuge, H. (1990). Bubble formation at a single orifice in highly viscous liquids. Journal of Chemical Engineering of Japan, 23(2), 160-165. Retrieved from doi: 10.1252/jcej.23.160

Vinhas, P. A. M. (2015). Estudo do movimento da bolha de taylor em coluna vertical e ligeiramente inclinável de líquido estagnado utilizando uma técnica de visualização. Tese de doutorado, Universidade Federal do Rio de Janeiro, Rio de Janeiro, RJ, Brasil.

Weisman, J., \& Kang, S. Y. (1981). Flow pattern transitions in vertical and upwardly inclined lines. International Journal of Multiphase Flow, 7(3), 271-291. Retrieved from doi: 10.1016/03019322(81)90022-7

Zukoski, E. E. (1966). Influence of viscosity, surface tension, and inclination angle on motion of long bubbles in closed tubes. Journal of Fluid Mechanics, 25(04), 821-837. Retrieved from doi: 10.1017/S0022112066000442 